\title{
The Status quo, challenges and countermeasures of nuclear power development in China
}

\author{
Mengyuan Dai ${ }^{1, a}$ \\ ${ }^{1}$ School of Business, Hohai University, Nanjing, China
}

\begin{abstract}
In China, the proportion of coal in the energy structure is high, and the utilization of coal will cause carbon emission and environmental pollution. Therefore, the adjustment of energy structure and the utilization of new energy has become the focus of China's efforts to comprehensively promote the green development of energy. At present, developing nuclear power has become a key way to break through the bottleneck of new energy power supply and realize economic and ecological benefits. Based on the current situation and existing problems of China's nuclear power industry, this paper puts forward the challenges and measures for the future development of nuclear power.
\end{abstract}

\section{INTRODUCTION}

The new energy security strategy of "Four Revolutions and One Cooperation" defines the two important directions of China's energy development as energy security and green development of energy. People's yearning for a better life is growing day by day, and advocating low carbon and environmental protection has become the consensus of the whole society. In the process of generating electricity, Nuclear power do not produce air pollutants such as sulfur dioxide, nitrogen oxide and soot, carbon dioxide emissions are far lower than thermal power. Nuclear power, as a kind of high-density energy, has a large capacity and can effectively guarantee the quality of power. To advance nuclear power construction in an orderly and steady way is the basic strategy of China, and to develop nuclear power safely and efficiently is the inevitable choice for us to enter the era of clean energy in an all-round way. In recent years, the world has mainly carried out research on the third and fourth generation nuclear power technology. The third-generation nuclear power technology is becoming more and more mature, with great improvement in economy and safety. This has laid a technological foundation for the development of China's nuclear energy industry. Therefore, the development of nuclear power has become a key way to break through the bottleneck of new energy power supply and achieve economic and ecological benefits.

\section{CHINA'S ENERGY SECURITY}

Energy is the basis and driving force for the progress of human civilization and is crucial to economic and social development. General Secretary Xi Jinping attaches great importance to China's energy development, puts forward a new energy security strategy of "four revolutions and one cooperation", opens up a new path of energy development with Chinese characteristics, and defines the two important directions of China's energy development as energy security and green development of energy.

\subsection{Energy Security}

Ensuring energy security is the basis and premise of developing nuclear power industry in China. From 2000 to 2019, China's energy self-sufficiency rate showed an overall downward trend, from $94.29 \%$ in 2000 to $81.52 \%$ in 2019, indicating that China's use of imported energy has increased and its dependence on external energy has gradually increased.

On the one hand, the degree to which a country or region's energy production meets its consumption is an important factor affecting the country's control of energy security. Countries with low energy self-sufficiency have higher foreign trade dependency of energy. But excessive dependence on energy imports will lead to increased economic uncertainty. Domestic economy and society are easily affected by war, political relations and international situation. On the other hand, with the rapid economic and social development, China's demand for energy has greatly increased, and it is impossible for the country to fully achieve energy self-sufficiency and meet the domestic production demand by importing energy. However, in order to reduce the political risks associated with imported energy, China should expand multi-directional energy import channels and disperse energy import risks. At present, China's maritime energy import channels mainly include the Middle East shipping routes, Africa shipping routes, Southeast Asia shipping routes and South America shipping routes. Among them, Middle East shipping routes pass through the Strait of Hormuz, and the actual control of the Strait of Hormuz is

avelpro7533@163.com 
controlled by the Iranian navy. If the United States or the international community imposes sanctions against economic or nuclear research and development on Iran, Iran may block the Strait of Hormuz and cut off $90 \%$ of the crude oil transportation in the Gulf region. Africa's shipping lanes pass through the Gulf of Aden, making them vulnerable to Somali pirates. The countries around the Strait of Malacca negotiated the Regional Maritime Security Plan with the United States, which was intended to contain China's maritime trade lifeline. The pipeline transportation in China mainly leads to Myanmar, Russia and Brazil, but the pipeline transportation is greatly affected by geography and is not suitable for long-distance transportation. Therefore, China should rely on the "The Belt Road" Maritime-Continental Silk Road to explore and develop multiple sea and land energy transport channels and build a global energy import network.

\subsection{Green Development of Energy}

Green development of energy is another major direction of energy development in China. Coal and oil are the two main energy sources consumed in China. However, coal and oil produce large amounts of harmful gases such as carbon dioxide and sulfur dioxide during the production process, which pollute the ecological environment. To achieve green development in the energy sector, China must adjust the stock, reduce low quality and ineffective supply, optimize increment, and raise the overall efficiency of the supply system. On the basis of promoting the reduction of excess capacity in the coal and coal power industries, China will increase quality and incremental supply and promote the consumption of renewable energy, so as to speed up the building of a clean, low-carbon, safe and efficient energy system and improve the quality of the energy supply system. In addition, the proportion of coal in China's energy structure is too high, while the proportion of other clean energy sources such as electricity and natural gas is too low. The use of coal will cause carbon emissions, resulting in ecological environmental pollution, which is contrary to China's carbon neutral development goal. Therefore, adjusting the energy structure is the focus of China's efforts to comprehensively promote green development.

\section{NUCLEAR POWER DEVELOPMENT}

\subsection{Development of international nuclear power industry}

Since June 27, 1954, when the Soviet Union built the world's first Obninsk nuclear power plant and used nuclear power for the first time, the world's nuclear power has been developing for more than 60 years. Worldwide, the development of nuclear power mainly goes through four stages: initial stage, outbreak stage, low tide stage and recovery stage.

(1)Initial period (1954-1965)

During this period, a total of 38 units were put into operation in the world, and several major nuclear countries built their own early prototype reactors, namely the first-generation nuclear power technology.

(2)The outbreak period (1966-1980)

With the rapid economic development of the United States, Japan, Europe and other developed countries, there is a huge demand for electricity supply. In 1973, the first oil crisis broke out, and nuclear power entered the stage of explosive growth worldwide due to its advantages of operating economy and energy independence. During this period, a total of 242 units were put into operation, all using second-generation nuclear technology. By the end of 1980, nearly 300 nuclear power units were in operation all over the world, with a total installed capacity of 180 million kW. From 1966 to 1980, the annual growth rate of nuclear power capacity reached $26 \%$.

(3)Low tide period (1981-2000)

The economic development of western countries slowed down due to the impact of the second oil crisis in 1979, which caused the demand for electricity to fall; However, the occurrence of two major accidents, the Three Mile Island Nuclear Power Plant in the United States in 1979 and the Chernobyl Nuclear Power Plant in the former Soviet Union in 1986, directly led to the stagnation of the development of nuclear power around the world, and the safety and economy of nuclear power were re-evaluated. Western countries adjust the nuclear power policy, the development speed slowed down obviously; With countries such as China, India and South Korea starting to step up, Asia has become the new growth pole for the nuclear industry.

(4)Recovery period (2001-present)

After entering the new century, reducing greenhouse gas emissions has become a difficult problem facing all countries in the world; At the same time, the progress of electrification of the whole society and the sharp fluctuation of energy supply have promoted the growth of power demand again. As a result, countries around the world turned to nuclear power again positive attitude, nuclear power industry began to recover. Although the Fukushima nuclear accident in Japan in March 2011 aroused doubts about the safety of nuclear power in the world, most countries still choose to continue to develop nuclear power because of the indispensable role of nuclear power in the world's energy supply. The Fukushima nuclear accident in Japan did not fundamentally change the situation of developing nuclear power around the world, but put forward more stringent requirements for the design and operation safety of nuclear power units.

\subsection{The development history and Status quo in China}

Beginning in the 1970s, nuclear energy in China began to take off. In 1985, the first self-designed and constructed Qinshan Nuclear Power Plant broke ground and was connected to the grid on December 15, 1991.Since then, there have been three major stages of nuclear power development. After 2000, China's nuclear power industry has entered a moderate development stage. Under the guidance of the government, Zhejiang Qinshan Phase II, Guangdong Lingao Phase I, and Qinshan Phase III were 
completed one after another. From 2006 to 2011, the nuclear power industry entered an active development stage. The first two stages are the rapid rise and development of China's nuclear power industry before the Fukushima event. After the Fukushima nuclear energy leak in Japan, China's nuclear energy development has stalled. The Fifth Plenary Session of the 17th Central Committee of the Communist Party of China proposed the policy of "Ensuring the efficient development of nuclear energy under safe conditions". Since then, China's nuclear power industry has developed steadily. The proportion of China's nuclear energy installed capacity has shown a fluctuating upward trend, with China's nuclear energy installed capacity reaching 48.74 million kilowatts, accounting for $2.42 \%$ in 2019. Meanwhile, from the perspective of the spatial layout of the nuclear power industry, Chinese nuclear energy plants are mostly built in coastal areas, the nuclear power plants in Fujian, Guangdong, and Hainan are denser.

In conclusion, from the perspective of the history law of nuclear power development, power as the basis of social production and living, public opinion and other factors have little influence on the choice of whether to develop nuclear power, actual factors such as economic and social development are more important in the overall consideration, while the fundamental determinants of the nuclear power industry development lies in electricity demand and energy security.

\section{CHALLENGES AND MEASURES OF NUCLEAR POWER DEVELOPMENT IN CHINA}

A tsunami caused by a massive earthquake knocked out emergency generators at Japan's Fukushima Daiichi nuclear power plant in 2011, shutting down its cooling systems. The disastrous consequences of nuclear meltdowns, hydrogen explosions and leaks of nuclear material caused by the private Tokyo Electric Power Company (TEPCO) triggered a global anti-nuclear wave.

\subsection{Security problem}

Drawing lessons from the Fukushima incident, although there has not been a major accident in the development of nuclear power in China, we still need to pay attention to the safety issues in the development of nuclear power. China's nuclear power safety is currently facing two major challenges. The first is the location of nuclear power plants. China's coastal nuclear power plants are heavily constructed, while inland resources have not been effectively utilized, but the government has not yet made it clear whether nuclear power plants can be built inland. At present, the third-generation nuclear power technology should be tested and tried for many times, and the situation of inland areas should be comprehensively inspected, and the basic work of pre-construction safety survey should be done well. Second, China's current safety supervision system is not perfect. With the continuous expansion of nuclear power plants in China, it is more and more difficult to supervise the nuclear power industry. In order to solve this problem, China built a system of nuclear security policies and regulations, implemented the national nuclear security strategy, formulated medium-term and long-term plans for nuclear security and improved nuclear safety regulations and standards to ensure that nuclear safety management requires a level of high not of low, strict not lenient. Coordinate nuclear safety activities and ensure that all safety-related activities are protected by multiple barriers to enhance the capability of serious accident prevention and mitigation. Then, China has established a three-level radiation environment monitoring system at the national, provincial and municipal levels, and established a national radiation environment quality monitoring network, a supervisory monitoring network for the radiation environment around key nuclear facilities, and a nuclear and radiation emergency monitoring network, so as to achieve fully-covered and all-weather monitoring of the radiation environment, and comprehensively promote nuclear safety improvement actions. In addition, China has made unremitting efforts to strengthen the building of a nuclear security culture and established a public communication mechanism on nuclear security featuring supervision by the central government, leadership by local governments, participation by enterprises and public participation. China standardize and guide the thinking and behavior of the practitioners and encourage the public to participate extensively to create a sound atmosphere in which everyone has a responsibility and participation, and the whole industry and society work together to safeguard nuclear security.

\subsection{Technology problem}

Innovating nuclear power technology is the critical path to solving safety and cost problems. China is gradually realizing the independence of nuclear power standards and the nationalization of nuclear power equipment. First, the nuclear power industry has accelerated efforts to unify the standards and technical routes for nuclear island machinery and equipment, strengthened the formulation and revision of standards, established a dynamic management mechanism for nuclear power standards in the energy industry, and improved the quality of standards. Second, promote the implementation and application of nuclear power standards, actively improve the accreditation system for nuclear safety-related standards, improve the implementation feedback mechanism, and realize the virtuous cycle of standard implementation, feedback and improvement. Third, in the localization work, the development cycle of major equipment directly determines the construction schedule of the whole nuclear power project. The current approach is to combine the imported foreign advanced technology with the domestic localization force to focus on the breakthrough of the localization of key equipment. In the practice stage, localization work needs to rely on limited project construction to continuously verify the coordination ability of various departments of localization and test the effectiveness degree of localization of nuclear power industry. 


\subsection{Cost problem}

The cost problem is an important challenge for nuclear power enterprises. The construction cycle of nuclear power plants is long and the cost is high. After the completion of the nuclear power plant, equipment maintenance and other activities to ensure the safety of operation will produce safety costs, and the import of foreign nuclear power technology or management experience will also produce many import costs. The high cost directly affects the competitiveness of nuclear power in the electricity market and is not conducive to China's nuclear power export. In order to solve this problem, nuclear power enterprises should realize the whole-process management of all personnel and all elements, reduce redundant steps in the management process, improve the management level of the project construction process, and strengthen the quality control, schedule control and investment control.

\section{DISCUSSIONS}

Energy is the basis and driving force for the progress of human civilization and is crucial to economic and social development. The proportion of nuclear power in China is relatively low, and China have the advantage of developing the space of nuclear power and the technology of fusion and innovation. However, there are still three problems in the development of nuclear power in China: security, technology and cost. In order to expand the scale of nuclear power development in the future, further development can be sought from such aspects as improving nuclear safety culture, strengthening safety management and supervision, attaching importance to the construction of nuclear power industry chain, improving the level of nuclear power equipment localization, and encouraging independent innovation of nuclear power enterprises.

On the whole, China's nuclear power is developing steadily and soundly. Looking ahead to the new stage and journey, the Fifth Plenary Session of the 19th Central Committee of the Party made a systematic plan for economic and social development during the "14th Five-Year Plan" period and even 2035.Focusing on the overall strategy of the great rejuvenation of the nation and major changes unseen in a century, promoting high-quality energy development from a new starting point has a deeper connotation. In the future, the capability and level of the nuclear energy industry chain for coordinated development, balanced development, and sustainable development will be improved year by year. From the perspective of industrial layout, in areas where nuclear power is more concentrated, the site selection and construction of low and intermediate level solid waste disposal sites will accelerate. And the development of inland nuclear power will also be fully considered. In addition to the advantages of clean power generation, nuclear energy will also play an important role in industrial steam supply, seawater desalination, isotope production, refrigeration and so on. In the future, the nuclear power industry will be safer, more diversified and more efficient.

\section{References:}

1. Zhang S.L., Li Q. The Development and Scale of the Nuclear Power in China under the Restraint of Low Carbon. China Population, Resources and Environment, 2015,25(06):47-52.

2. Wang Z.Y., Liu M. Review and Suggestions on Nuclear Power Industry in China [J]. China Mining Magazine,2020,29(S1):5-11.

3. Zhao C.K. Development Status and Outlook for Nuclear Power in China [J]. Nuclear Power Engineering,2018,39(05):1-3.

4. Zhou J.Y.,et al., Status and Trend of China's Nuclear Power Development in the Post-Fukushima Era [J]. Future and Development, 2015,39(09):42-46.

5. Yang Y.H., et al., Analysis on the development of localization of China's nuclear power industry [J]. Science Technology and Industry, 2020,20(05):113-118.

6. Han Z.H., Chen F.B. and Qin X.Y. Analysis on Nuclear Power and Renewable Energy Coordinated Development in future [J]. Shanghai Energy Conservation, 2020(12):1374-1380. 